\title{
Multi-choice opinion dynamics model based on Latané theory
}

\author{
Przemysław Bańcerowski and Krzysztof Malarz ${ }^{\mathrm{a}}$ \\ AGH University of Science and Technology, Faculty of Physics and Applied Computer Science, al. Mickiewicza 30, \\ 30-059 Krakow, Poland
}

Received 5 September 2018 / Received in final form 16 July 2019

Published online 2 October 2019

(C) The Author(s) 2019. This article is published with open access at Springerlink.com

\begin{abstract}
In this paper Nowak-Szamrej-Latané model is reconsidered. This computerised model of opinion formation bases on Latané theory of social impact. We modify this model to allow for multi (more than two) opinions. With computer simulations we show that in the modified model the signatures of order/disorder phase transition are still observed. The transition may be observed in the average fraction of actors sharing the $i$ th opinion, its variation and also average number of clusters of actors with the same opinion and the average size of the largest cluster of actors sharing the same opinion. Also an influence of model control parameters on simulation results is shortly reviewed. For a homogeneous society with identical actors' supportiveness and persuasiveness the critical social temperature $T_{C}$ decreases with an increase of the number of available opinions $K$ from $T_{C}=6.1(K=2)$ via $4.7,4.1$ to $T_{C}=3.6$ for $K=3,4,5$, respectively. The social temperature plays a role of a standard Boltzmann distribution parameter containing social impact as the equivalent of energy or one may think about it just as on a noise parameter.
\end{abstract}

\section{Introduction}

Simulations of opinion dynamics [1] are core subject of sociophysics [2,3], an interdisciplinary field of research in complex systems directly connected to computational sociology. Numerous examples of such research are published in interdisciplinary sections of physical journals [4-11], and in journals devoted to computational sociology [12-18]. The models of opinions dynamics deals with binary (or Boolean), Ising-like $[19,20]$ variables, corresponding to two-states models of opinions [21-24] or multi-state, but still discrete state opinions models $[7,25]$ or discrete vector-like variables [8]. The second group of models deals with continuous opinions [12,14,15,26-34].

Another classification of opinion dynamics models may be based on geometry of underlying network of connections among actors. Basing on this criteria we can deal with continuous (plane-like) $[6,13,35,36]$ or discrete geometry. The later may be divided into additional subgroups, with regular lattices [23-25,27,37] or complex networks [9,21,38-43].

The last classification includes system dynamics in terms of time evolution of the system, which again may occurring in discrete or in continuous time.

Assumed scheme of system representation force choosing the most adequate numerical technique for computer simulation of the system, including solving set of differential equations [44] (continuous space of opinions, continuous geometry and continuous time) or cellular

\footnotetext{
${ }^{\mathrm{a}}$ e-mail: malarz@agh.edu.pl
}

automata technique [45-49] (discrete space of opinions, discrete time and discrete geometry).

The topic of public opinion formation and its dynamics still brings vivid interest of researches (see Refs. [50-55] for the most recent papers).

In this paper Nowak-Szamrej-Latané model is reconsidered [56]. We propose multi-choice opinion dynamics model based on Latané [57-59] theory. With computer simulation we show that in the system with the long-range interactions among actors and more than two opinions the order-disorder phase transition is also observed.

\subsection{Theory of social impact}

The mathematical model being the foundation of this work relies on Latané social impact theory [57-59] and its computerised version proposed by Nowak et al. [56]. This approach for binary opinions and possible charismatic leader localised in the system centre has been thoroughly explored in Hołyst, Kacperski and Schweitzer papers [10,11] (see Ref. [60] for review).

Latané assumes that people are social animals and in their natural environment (society) they influence each other. These interactions do not have to be intentional. Under this assumption we understand all interactions among people. Persuasion, joke, sharing emotions and feelings - all of these can affect others. Latané describes these interactions as social impact.

The theory of social impact bases on three fundamental principles: (i) social force, (ii) psycho-social law and (iii) multiplication/division of impact. 


\subsubsection{Social force}

The social force principle [59] says that social impact $I$ on $i$ th actors is a function of the product of strength $S$, immediacy $J$, and the number of sources $N$

$$
I=\mathcal{F}(S J N) .
$$

The strength of influence is the intensity, power or importance of the source of influence. This concept may reflect socio-economical status of the one that affects on our opinion, his/her age, prestige or position in the society.

The immediacy determines the relationship between the source and the goal of influence. This may mean closeness in the social relationship, lack of communication barriers and ease of communication among actors.

Latané called this principle 'a bulb theory of social relations'. According to this analogy the social impact plays a role of illuminance. The illuminance depends on

- the power of the bulb (physicists prefer to think about bulb's luminous flux) - equivalent of the strength of impact

- the distance from sources (bulbs) - equivalent of the immediacy

- and the number of bulbs - equivalent of the number of people.

\subsubsection{Psycho-social law}

The formula proposed by Latané is fitted to the data of Gerard et al. [61] (which were obtained in a repetition of the famous Asch [62] experiment) and Milgram et al. [63] experiments:

$$
I \propto S N^{\beta},
$$

where $N$ is the number of people exerting the impact, $S$ is a strength of impact and $0<\beta<1$ is the scaling exponent.

This means that each next actor $j$ sharing the same opinion as actor $i$ exerts the lower impact on the $i$ th actor. This formula has been independently confirmed experimentally by Latané [57].

\subsubsection{Multiplication/division of impact}

The lecture for single student influence his/her much more the same lecture given for hundred of students. In the latter case, the impact of lecture is roughly equally divided among all listeners [64]. For this issue Latané proposes

$$
I \propto S N^{-\gamma}
$$

where the scaling exponent $0<\gamma<1$.

Latané confirmed the legitimacy of using formula (3) with research on the bystander effect $[65,66]$ carried out jointly with Darley [64]. This experiment was repeated many times by various researchers. Latané and Nida [58] collected the results of nearly one hundred of such studies and validated equation (3).

\subsubsection{The limitations of the theory}

The main limitation of the social impact theory lies in treating people as totally passive. The second trouble is the absence of dynamics in the model. These issues have been solved by Nowak et al. [56] in the computerised version of Latané model.

\section{Model}

Every actor at position $i$ is characterised by his/her discrete opinion $\xi_{i}$, his/her persuasiveness $\left(0 \leq p_{i} \leq 1\right)$ and his/her supportiveness $\left(0 \leq s_{i} \leq 1\right)$. Parameter $p_{i}$ describes the intensity of persuasion to change the opinion by actor $i$ from a person with opinion different than $\xi_{i}$, while $s_{i}$ describes the intensity of supporting people with the same views.

\subsection{Two opinions $(K=2)$}

For two opinions one can assume integer values of $\xi_{i} \in$ $\{-1,+1\}$. For evaluation of social impact $I_{i}$ on actor at position $i$ one can apply formula proposed in reference [60]:

$$
\begin{aligned}
I_{i}(t)= & \mathcal{J}_{P}\left(\sum_{j=1}^{N} \frac{q\left(p_{j}\right)}{g\left(d_{i, j}\right)}\left[1-\xi_{i}(t) \xi_{j}(t)\right]\right) \\
& -\mathcal{J}_{S}\left(\sum_{j=1}^{N} \frac{q\left(s_{j}\right)}{g\left(d_{i, j}\right)}\left[1+\xi_{i}(t) \xi_{j}(t)\right]\right),
\end{aligned}
$$

where $\mathcal{J}_{P}(\cdot), \mathcal{J}_{S}(\cdot), q(\cdot), g(\cdot)$ stand for scaling functions and $d_{i, j}$ is Euclidean distance between sites $i$ and $j$. The system dynamics may be governed by heat-bath-like dynamics [11], i.e.:

$$
\xi_{i}(t+1)=\left\{\begin{array}{rr}
\xi_{i}(t) \quad \text { with probability } \frac{\exp \left(\frac{-I_{i}(t)}{T}\right)}{2 \cosh \left(\frac{I_{i}(t)}{T}\right)}, \\
-\xi_{i}(t) \quad \text { with probability } \frac{\exp \left(\frac{I_{i}(t)}{T}\right)}{2 \cosh \left(\frac{I_{i}(t)}{T}\right)},
\end{array}\right.
$$

where $T$ is a noise parameter (social temperature $[67,68]$ ). The meaning of the social temperature $T$ parameter is not different to the meaning of temperature in Ising $[19,20]$ or majority-voter model [69]. Namely, for $T=0$ the system is fully deterministic while for $T \rightarrow \infty$ the actors decisions (and accepted opinion) are random.

For $T=0$ the rule (5) may be reduced to fully deterministic rule [11]

$$
\xi_{i}(t+1)=\operatorname{sgn}\left(I_{i}(t)\right)
$$

as $I_{i}(t)=0$ is practically impossible to occur. 

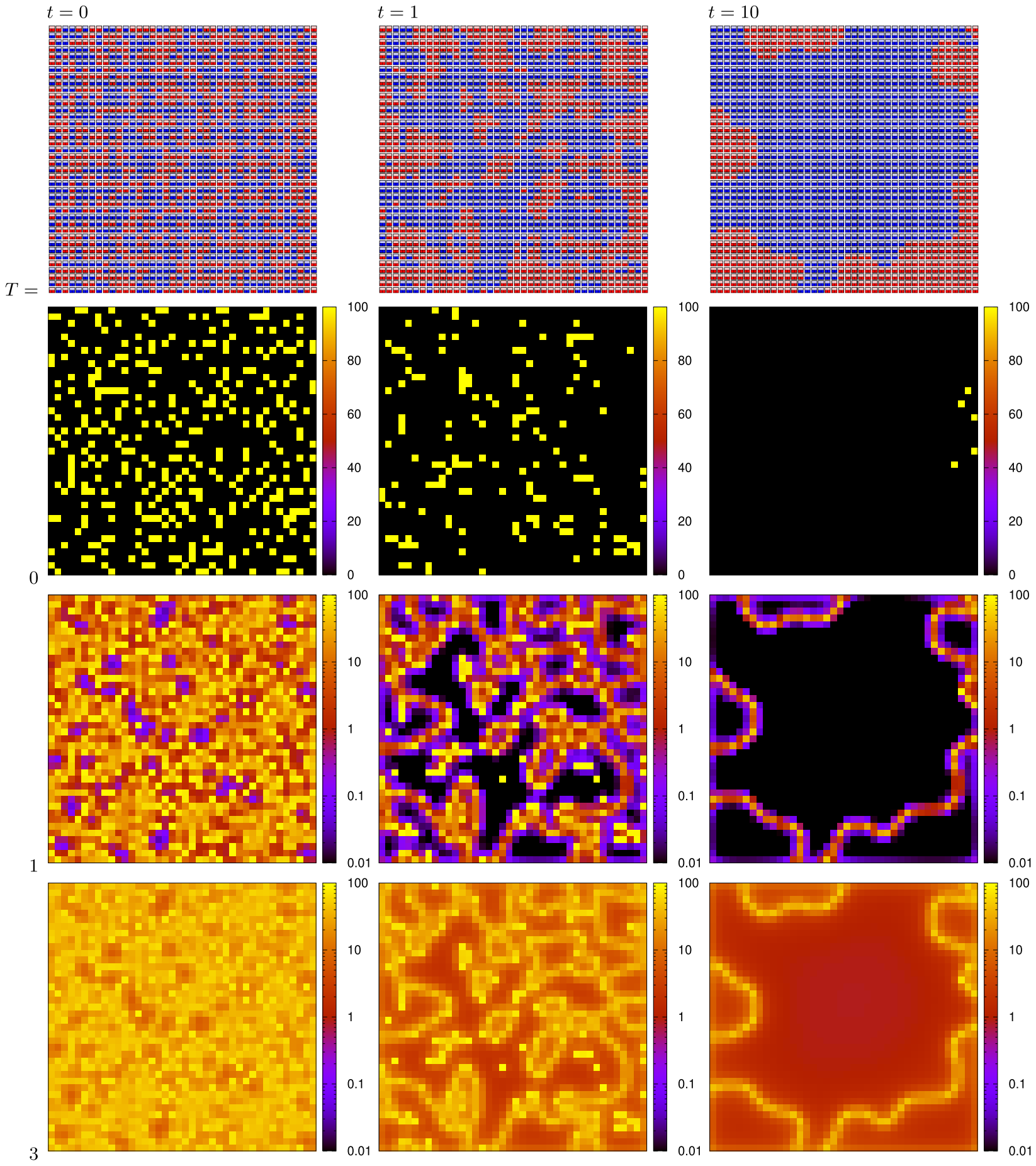

Fig. 1. In the top line the snapshots from simulation of the system containing $L^{2}=40^{2}$ sites and $K=2$ are presented. The blue and red colours correspond to various actors opinions $\left(\xi_{i} \in\{-1,+1\}\right)$. The subsequent columns correspond to time steps $t=0$, 1,10 , respectively. In subsequent rows the probabilities of changing opinion $\mathcal{P}_{i}$ associated with sites $i$ and for social temperature $T=0,1$ and 3 are presented. $\forall i: p_{i}=s_{i}=0.5, \alpha=3[70]$.

\subsection{Three and more opinions $(K>2)$}

For multi-state space of opinions we do not assign numeric values to opinions

$$
\xi_{i} \in\left\{\Xi_{1}, \Xi_{2}, \ldots, \Xi_{K}\right\}
$$

where $K$ is the number of available opinions. We rather prefer to think about various 'colours' of opinions, or about $K$ orthogonal versors in $K$-dimensional vector space. Also we propose some modifications of equation (4). We propose to separate the social impact on actor $i$ from actors $j$ sharing opinion of actor 


$$
\begin{aligned}
& i\left(\xi_{j}=\xi_{i}\right) \\
& \quad I_{i, k}(t)=4 \mathcal{J}_{s}\left(\sum_{j=1}^{N} \frac{q\left(s_{j}\right)}{g\left(d_{i, j}\right)} \delta\left(\Xi_{k}, \xi_{j}(t)\right) \delta\left(\xi_{j}(t), \xi_{i}(t)\right)\right)
\end{aligned}
$$

and all other actors having different $K-1$ opinions $\left(\xi_{j} \neq \xi_{i}\right)$

$I_{i, k}(t)=4 \mathcal{J}_{p}\left(\sum_{j=1}^{N} \frac{q\left(p_{j}\right)}{g\left(d_{i, j}\right)} \delta\left(\Xi_{k}, \xi_{j}(t)\right)\left[1-\delta\left(\xi_{j}(t), \xi_{i}(t)\right)\right]\right)$,

where $1 \leq k \leq K$ enumerates the opinions and Kronecker's delta $\delta(x, y)=1$ if $x=y$ and zero otherwise. The factor of four in equation (8) guaranties exactly the same impact on actor $i$ as calculated basing on equation (4) for $K=2$.

Please note, that terms $\left[1 \pm \xi_{i}(t) \xi_{j}(t)\right]$ from equation (4) are missing in equation (8). In equation (4) term [1 $\xi_{i}(t) \xi_{j}(t)$ ] vanishes when actors $i$ and $j$ have the same opinion $\left(\xi_{i}(t) \xi_{j}(t)=+1\right)$, which means that the term is active for actors' pairs having different opinions - the persuasion stage takes place. Term $\left[1+\xi_{i}(t) \xi_{j}(t)\right]$ vanishes when actors $i$ and $j$ have different opinion $\left(\xi_{i}(t) \xi_{j}(t)=\right.$ -1 ), which means that the term is active for actors' pairs having common opinions - the support stage takes place. When we do not assign numeric values for $\xi_{i}$ (as we do for $K>2$ ) the equation (8a) is applied if actors $i$ and $j$ have the same opinion, and we use equation (8b) to calculate $K-1$ influences $I_{i, k}$ on $i$ th actor from actors $j$ having $K-1$ opinions different from the opinion of $i$ th actor.

The calculated social impacts $I_{i, k}(t)$ influence the $i$ th actor opinion $\xi_{i}(t+1)$ at the subsequent time step. For $T=0$ this opinion is determined by this opinion $k$ which believers exert the largest social impact on $i$ th actor

$$
\begin{aligned}
\xi_{i}(t+1) & =\Xi_{k} \\
\Longleftrightarrow I_{i, k}(t) & =\max \left(I_{i, 1}(t), I_{i, 2}(t), \ldots, I_{i, K}(t)\right) .
\end{aligned}
$$

For finite values of social temperature $T>0$ we apply the Boltzmann choice

$$
p_{i, k}(t)=\exp \left(\frac{I_{i, k}(t)}{T}\right)
$$

which yield probabilities

$$
P_{i, k}(t)=\frac{p_{i, k}(t)}{\sum_{j=1}^{K} p_{i, j}(t)}
$$

of choosing by $i$ th actor in the next time step $k$ th opinion:

$$
\xi_{i}(t+1)=\Xi_{k} \text {, with probability } P_{i, k}(t) .
$$

The form of dependence (10) in statistics and economy is called logit function $[71,72]$.

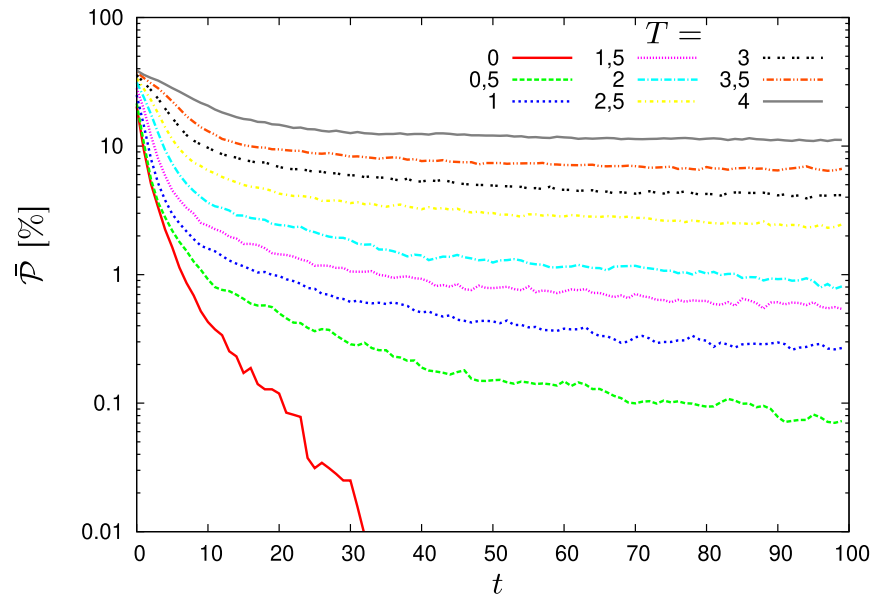

Fig. 2. The time evolution of the average changing opinion probability $\overline{\mathcal{P}}[\%]$ for various values of social temperatures $T$ [70].

We assume identity function for scaling functions $\mathcal{J}_{S}(x) \equiv x, \mathcal{J}_{P}(x) \equiv x, q(x) \equiv x$. The distance scaling function should be an increasing function of its argument. Here, we assume the distance scaling function as

$$
g(x)=1+x^{\alpha},
$$

what ensures non-zero values $g(0)=1$ of denominator for self-supportivenees in equation (8a). The exponent $\alpha$ is an arbitrary quantity which characterise the long-range interaction among actors. For small values of $\alpha$ (for instance for $\alpha=2$ ) we assume good communication among actors, well access to information in the society and good exchange of information. In contrary, for larger values of $\alpha$ (for instance for $\alpha=6$ ) discussion and information exchange takes place only in the actors' nearest neighbourhood.

Newly evaluated opinions are applied synchronously to all actors.

The simulations are carried out on square lattice of linear size $L=40$ with open boundary conditions. We assume identical values of supportivenees and persuasiveness for all actors $\forall i: s_{i}=p_{i}=0.5$. We set exponent $\alpha=3$ in the distance scaling function (13).

The web application allowing for direct observation of the system evolution is available at http://www.zis.agh. edu.pl/app/MSc/Przemyslaw_Bancerowski/. The short manual for this application is available in Appendix A.

\section{Results}

\subsection{Two opinions}

\subsubsection{Influence of the model parameters on opinion dynamics}

To understand better the system time evolution the maps of probabilities $\mathcal{P}_{i}$ of opinion changes at sites $i$ (for $K=2$ ) are presented in Figure 1. The snapshots of system states at $t=0,1$ and 10 are presented in the first row of Figure 1. 

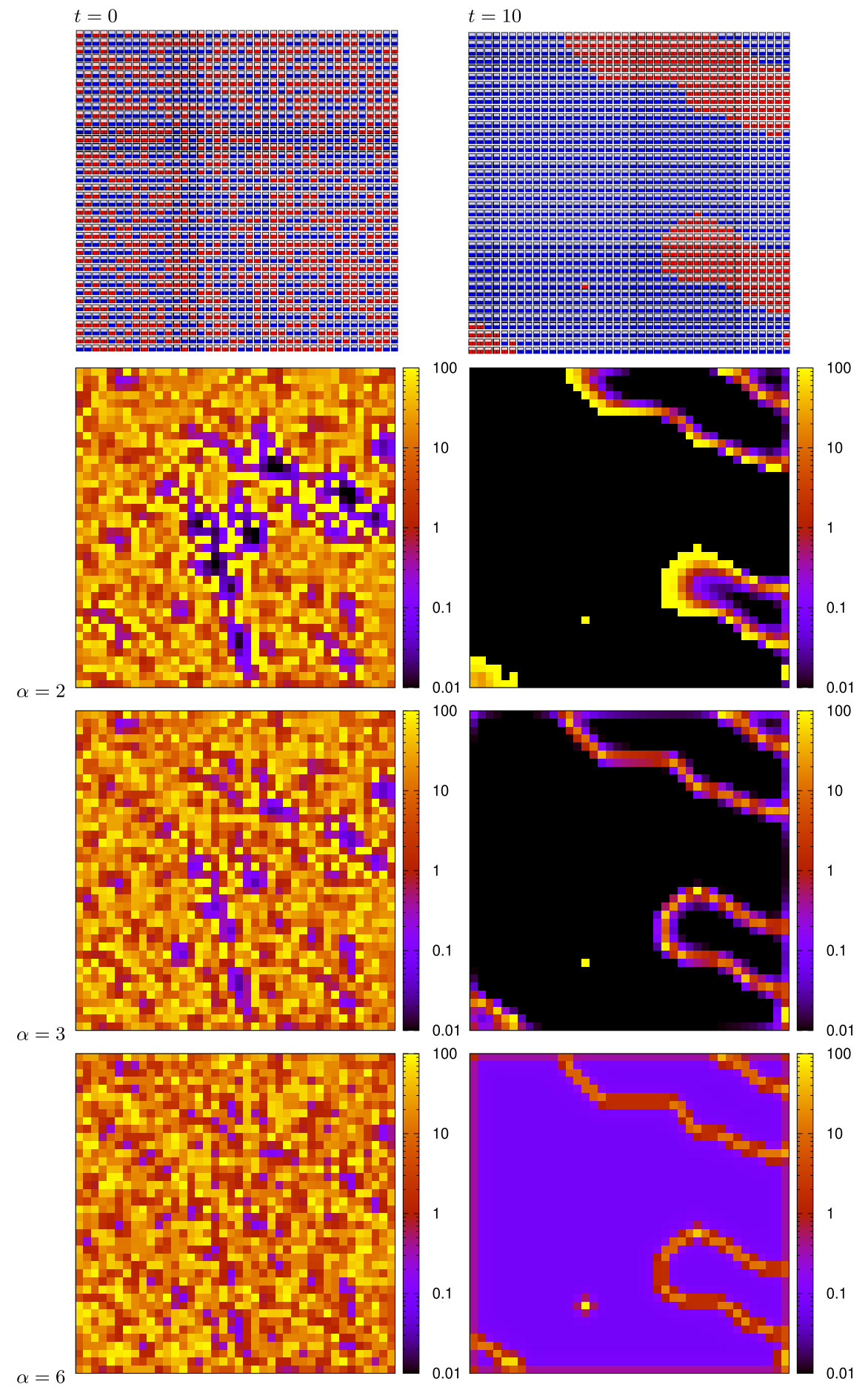

Fig. 3. The maps of probabilities of opinion changes $\mathcal{P}_{i}[\%]$ for social temperature $T=1$ at the initial random distribution of opinions $(t=0)$ and after ten time steps of simulation $(t=10)$ and for various values of exponent $\alpha=2,3$ and 6 . In the first row the snapshots from simulations indicating the spinsons opinions for $t=0$ (first column) and $t=10$ (second column) are presented [70]. The blue and red colours correspond to various actors opinions $\left(\xi_{i} \in\{-1,+1\}\right)$.

The corresponding to these states probabilities of opinion changing (flipping) for social temperatures $T=0,1$ and 3 are presented in the second, third and fourth row of Figure 1, respectively. For $T=0$ (the second row) the system is fully deterministic and $\forall i: \mathcal{P}_{i} \in\{0,1\}$. For long enough times of evolution the system reaches the nearly-steady state (with single spinsons ${ }^{1}$ going to change their minds) and clearly defined borders between groups

\footnotetext{
1 The term 'spinson' comes from merging words 'spin' and 'person' and it describes actor who may have only two opinions. The term was introduced in 2013 by Nyczka and Sznajd-Weron [5].
} 
(clusters) of spinsons with different opinions. The static picture of the system is also observed for $T>0$, with non-zero probabilities of changing opinions $\mathcal{P}_{i}$ for spinson $i$ located at the clusters borders.

In Figure 2 the time evolution of the spatial average of probabilities of opinion changes

$$
\overline{\mathcal{P}}=L^{-2} \sum_{i=1}^{L^{2}} \mathcal{P}_{i}
$$

is presented. The spatial average over $L^{2}$ sites is marked through this paper by a bar $\left({ }^{-}\right)$. For long enough times the average probabilities of opinion changes $\overline{\mathcal{P}}$ increases smoothly with increase of social temperature, reaching $\overline{\mathcal{P}} \approx 10 \%$ for $T=4$.

As expected, an increase the social temperature $T$ enhances the spinsons nonconformity, i.e. they are able to change their minds although social impact exerted on them by other members of the society with the same opinion. In the limit of infinite social temperature every actor chooses his/her opinion randomly, as

$$
\lim _{T \rightarrow \infty} p_{i, k}(t)=1 \quad \text { and } \quad \lim _{T \rightarrow \infty} P_{i, k}(t)=1 / K .
$$

In Figure 3 the maps of probabilities changes $\mathcal{P}_{i}$ are presented again. The first row shows the snapshots from simulations indicating the spinsons opinions for $t=0$ (first column) and $t=10$ (second column). The subsequent rows correspond to probabilities of opinion changes for various values of exponent $\alpha$ in the distance scaling function $g(x)$ [Eq. (13)] $-\alpha=2,3,6$ in the second, third and fourth row, respectively. The random initial configuration of opinions leads to random maps of $\mathcal{P}_{i}$. However, ten time steps of system relaxation allows for an observation of both: the spatial clusterization of spinsons shearing the same opinion and high probabilities of opinion changing at the borders of these clusters. Moreover, for high values of exponent $\alpha$ differences among the minimal and the maximal values of $\mathcal{P}_{i}$ are much smaller than for small values of $\alpha$.

Quantitatively these differences may be observed in Figure 4 for purely deterministic $(T=0)$ and nondeterministic $(T=1)$ cases. In principle, for $T>0$ the higher value of the exponent $\alpha$ leads to the higher value of $\overline{\mathcal{P}}$ which values saturate on the level $\overline{\mathcal{P}} \approx 1 \%$ after hundred simulation steps for $T=1$ and $\alpha>4$.

\subsubsection{Phase transition}

In Figure 5a the results on an average opinion

$$
\bar{\xi}(t) \equiv L^{-2} \sum_{i=1}^{L^{2}} \xi_{i}(t),
$$

for various values of the social temperature $T$ are presented. Similarly to the Ising model some signatures of the phase transition in the system may be observed. For low social temperature $\left(T<T_{C}\right)$ the system is in ordered
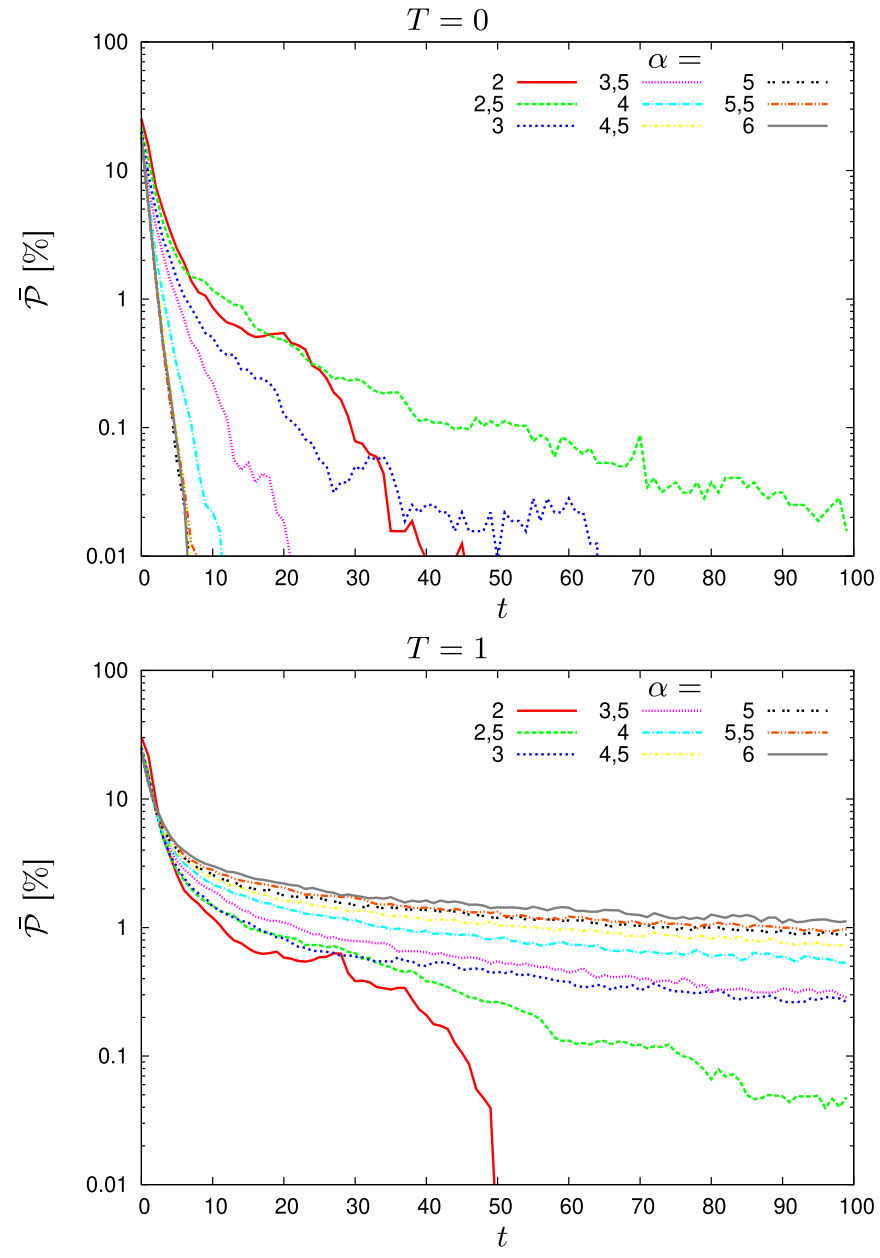

Fig. 4. The time evolution of the average probability $\overline{\mathcal{P}}[\%]$ of opinion changes for various values of the distance function scaling exponents $\alpha$ [70].

phase with majority of one (initially dominant) opinion. However, for high enough temperature $\left(T>T_{C}\right)$ the average opinion oscillates around $\bar{\xi}=0$.

In Figure $5 \mathrm{~b}$ an example of time evolution of the spatial average opinion in the system for $T \rightarrow T_{C}^{+}$is presented. Although the long-range interaction among actors is assumed, the time evolution $\bar{\xi}(t)$ is not different from 'magnetisation' evolution in the Ising model with characteristic 'magnetisation' switching between its positive and negative values above the Curie temperature.

In Figure 6a we plot the temporal average

$$
\langle\bar{\xi}\rangle \equiv \tau^{-1} \sum_{t=t_{0}}^{t_{M}} \bar{\xi}(t),
$$

for various temperatures $T$. The temporal average over $\tau$ times steps is marked through this paper by brackets $(\langle\cdots\rangle)$. Here, $\tau=5000-100$, i.e. the first hundred of time steps is excluded from the averaging procedure.

The ordered phase phase vanishes for $T>T_{C} \approx 6.1$. This critical value of $T_{C}$ coincidences nicely with a peak 
(a) $K=2$

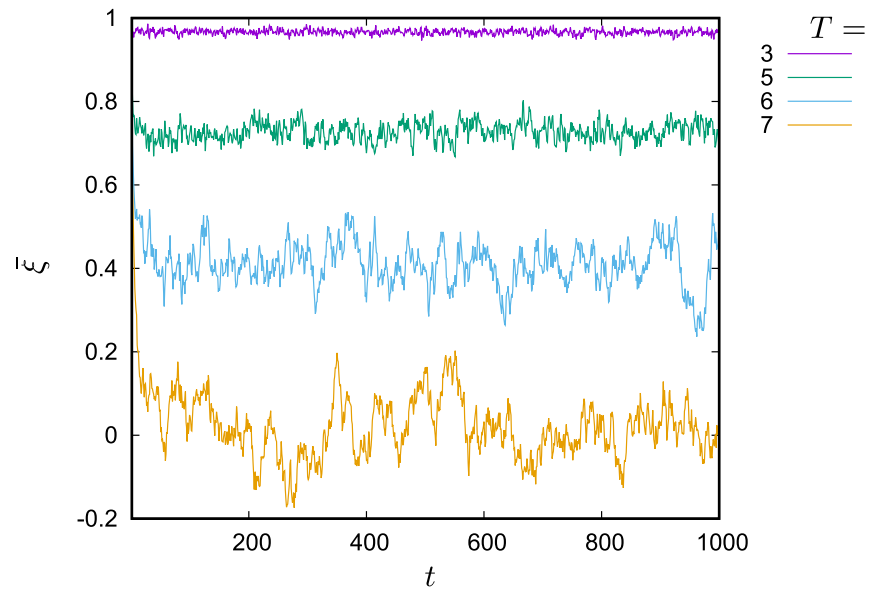

(b) $K=2, T=6.2>T_{C}$

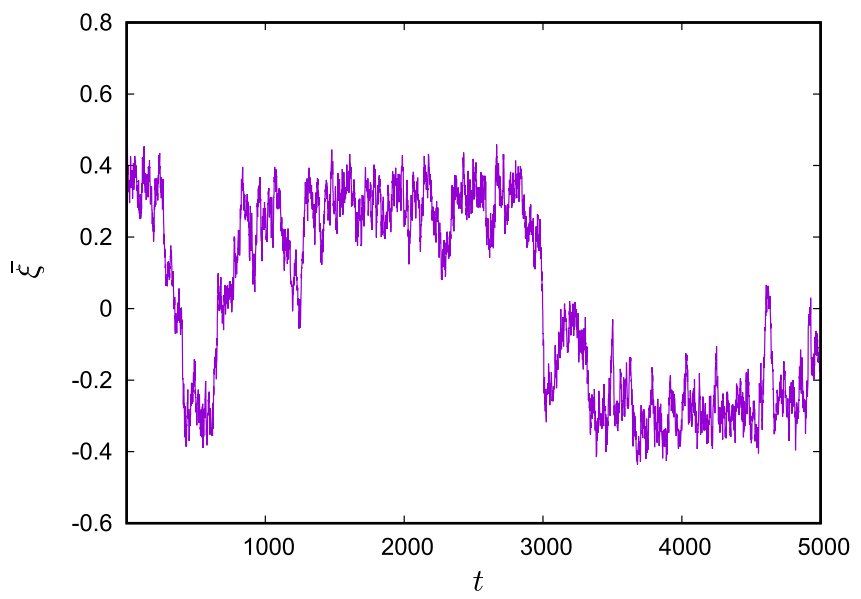

Fig. 5. The time evolution of average opinion $\bar{\xi}$ for $K=2$ opinions and various social temperatures T. $L=40, \alpha=3$, $\forall i: p_{i}=s_{i}=0.5[70]$.

of average opinion dispersion

$$
\sigma^{2}(\bar{\xi})=\left\langle\bar{\xi}^{2}\right\rangle-\langle\bar{\xi}\rangle^{2}
$$

as presented in Figure 6b. The values of $\sigma$ plays a role of static susceptibility $\chi$ in Ising-like systems. We confirm the earlier results indicating the phase transition in NowakSzamrej-Latané model for binary opinions [10].

In the next section we show that the above mentioned results are generic also when multi-opinions are available in the system.

\subsection{Three and more opinions}

As we mentioned in Section 2.2 for $K>2$ we do not assign numerical values to opinions $\xi_{i}$. Instead, we prefer to think about $K$ 'colours' $\Xi_{k=1, \cdots, K}$ of opinions (see Fig. 7 for snapshots from simulations presenting spatial distributions of opinions for $T=0,6$ and $K=3,6)$. This assumption does not allow for dealing with $\langle\bar{\xi}\rangle$ [Eq. (15)] and $\sigma(\bar{\xi})[\mathrm{Eq} .(16)]$ in order to identify the critical social temperature $T_{C}$. Thus for this purpose we propose to deal (a) $K=2$

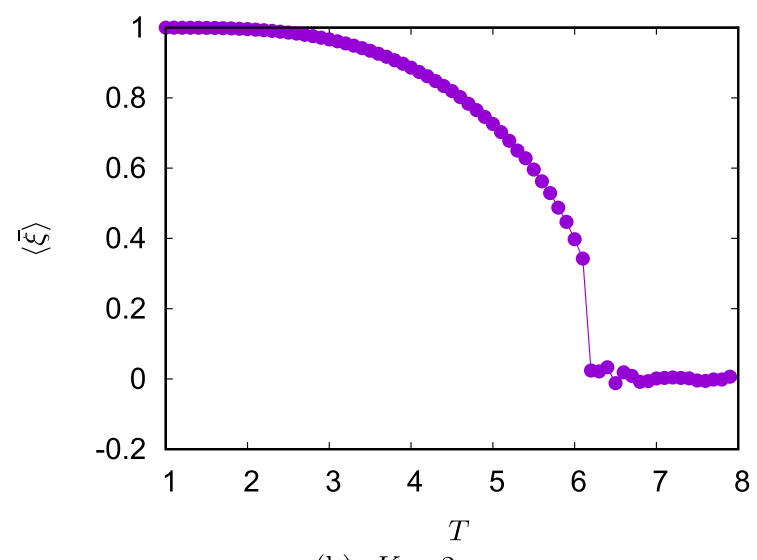

(b) $K=2$

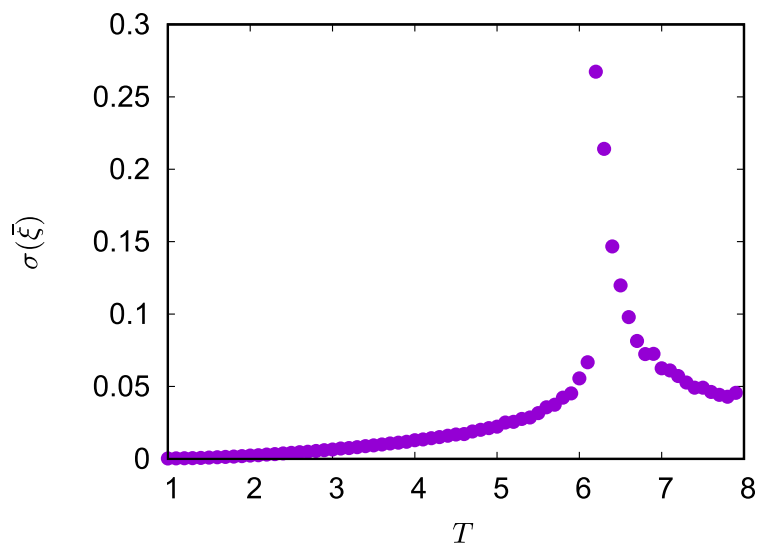

Fig. 6. The values of (a) average values of opinion $\langle\bar{\xi}\rangle$ and (b) its standard deviation $\sigma(\bar{\xi}) . L=40, \alpha=3, \forall i: p_{i}=s_{i}=$ 0.5 . The values of $\bar{\xi}$ are averaged over last $\tau=5000-100$ time steps [70].

with a fraction $\left\langle\bar{n}_{k}\right\rangle$ of actors sharing the $k$ th opinion and its standard deviation $\sigma\left(\bar{n}_{k}\right)$.

In Figures $8 \mathrm{a}-8 \mathrm{c}$ and Figures $8 \mathrm{~d}-8 \mathrm{f}$ we plot $\left\langle\bar{n}_{k}\right\rangle$ and $\sigma\left(\bar{n}_{k}\right)$ for $K=3,4,5$, respectively. As we can see in Figures $8 \mathrm{a}-8 \mathrm{c}$ the majority $\left\langle\bar{n}_{1}(t=0)\right\rangle$ of holders of opinion $\Xi_{1}$ vanishes with increasing the social temperature $T$. For critical social temperature $T \geq T_{C}$ all available opinions $\Xi_{1}, \cdots, \Xi_{K}$ in the system are equally occupied $\left(\left\langle\bar{n}_{1}\right\rangle=\left\langle\bar{n}_{2}\right\rangle=\left\langle\bar{n}_{3}\right\rangle \approx 33 \%\right.$ for $K=3$ and $\left\langle\bar{n}_{1}\right\rangle=\cdots=$ $\left\langle\bar{n}_{5}\right\rangle \approx 20 \%$ for $K=5$ ).

Again, vanishing of initially major opinion at $T=T_{C}$ coincidences nicely with maximal values of $\sigma\left(\bar{n}_{k}\right)$ as presented in Figures 8d-8f. Similar critical behaviour may be observed in thermal evolution of the size of the larger cluster of actors sharing the same opinion $\left\langle\overline{\mathcal{S}}_{\max }\right\rangle$ (see Figs. $8 \mathrm{~g}-8 \mathrm{i})$ and the total number $\langle\overline{\mathcal{C}}\rangle$ of clusters of actors sharing the same opinion (see Figs. $8 \mathrm{j}-81$ ). The increase of the number of clusters with increasing social temperature is also clearly visible in Figure 7.

\section{Discussion and conclusions}

In this paper we proposed multi-choice opinion dynamics model based on Latané theory. With computer simulation 
(a) $K=3, T=0$

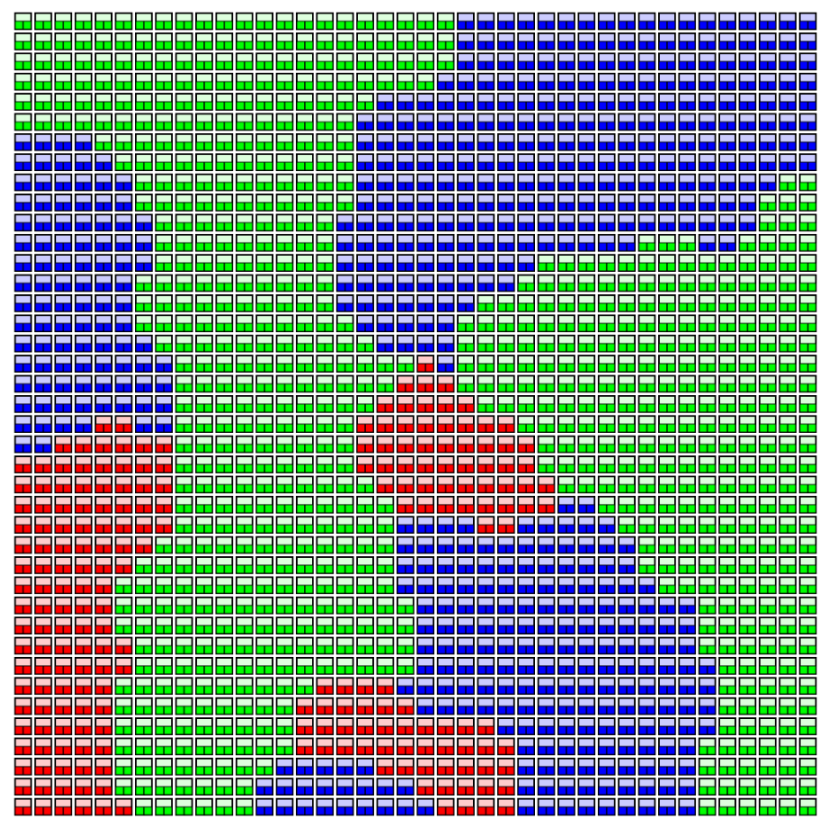

(c) $K=6, T=0$

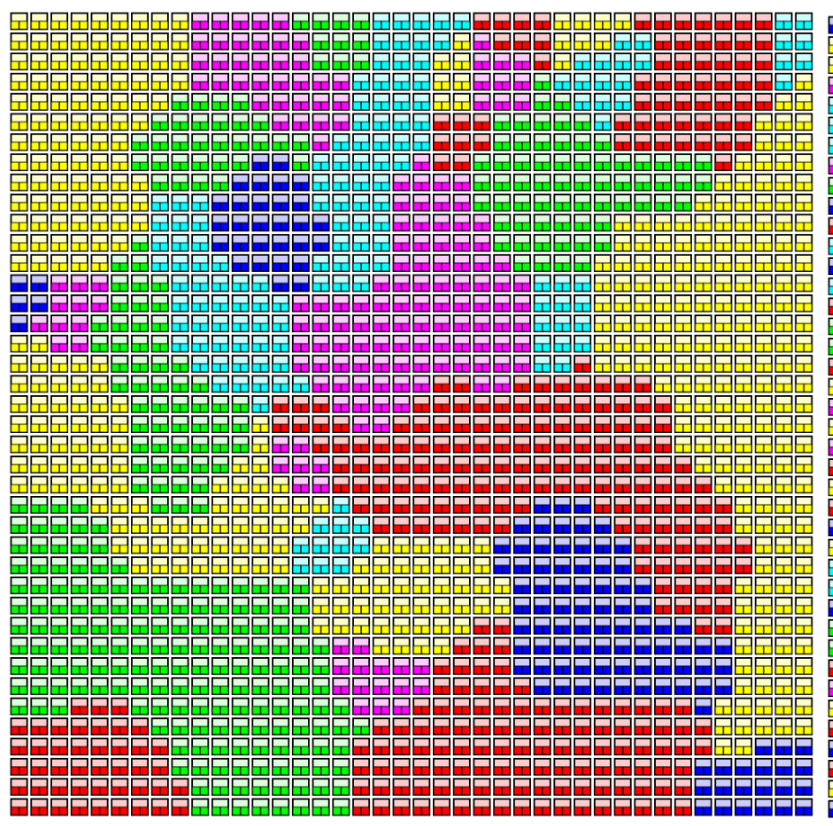

(b) $K=3, T=6$

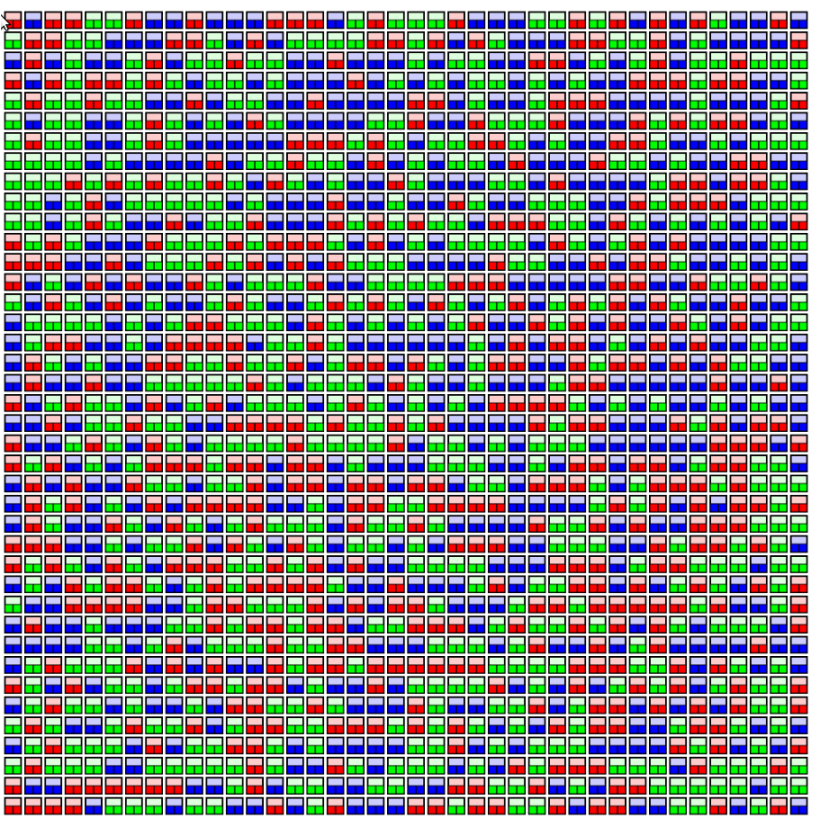

(d) $K=6, T=6$

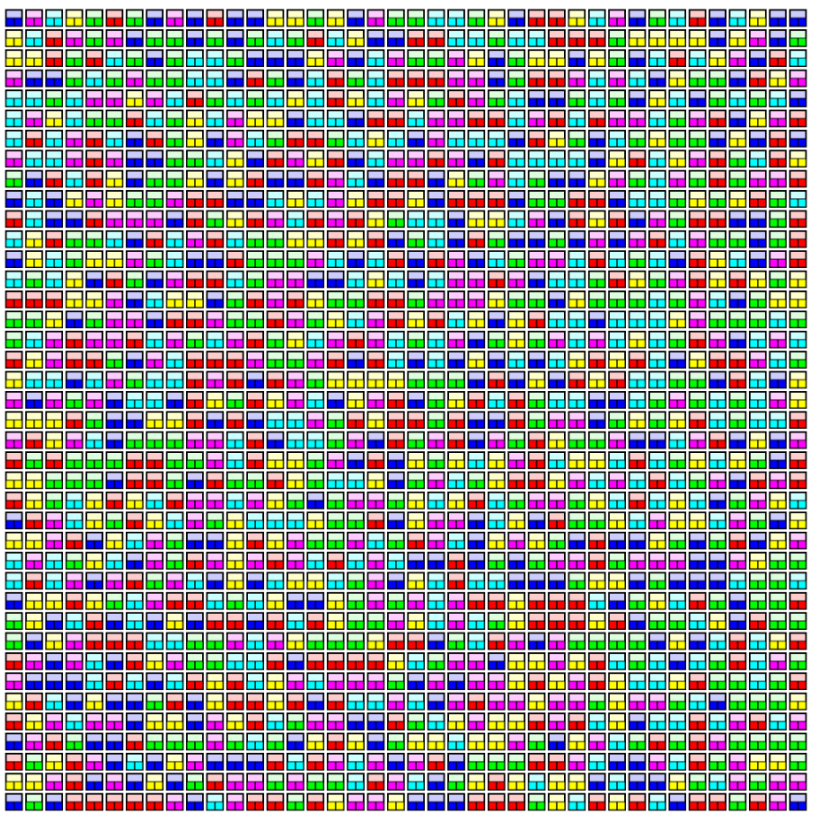

Fig. 7. The snapshots of opinions spatial distribution for social temperature $T=0$ (the first column) and $T=6>T_{C}$ (the second column) for various numbers of available opinions $K=3$ (the first row) and $K=6$ (the second row). The various colours correspond to various actors opinions $\left(\xi_{i} \in\left\{\Xi_{1}, \Xi_{2}, \ldots, \Xi_{K}\right\}\right)$.

we show, that for multi-opinion version of the NowakSzamrej-Latané model of opinion dynamics even without assigning numeric values for opinions we are able to observe phase transition similar to this occurring in two-state Ising-like models of opinion dynamics.

As we avoid signing a numerical values to possible opinions, we do not need to use the Likert-like scale [73] with possible Likert items as ' 1 = Strongly disagree', ' $2=$ Disagree', '3 = Neither agree nor disagree', ' $4=$ Agree' and
'5 = Strongly agree'. Likert scale falls within the ordinal level of measurement accordingly to the best known classification of scales of measurement by [74].

Instead of signing a numerical values to possible opinions we deal with $K$ 'colours' of opinions $\Xi_{1}, \Xi_{2}, \ldots, \Xi_{K}$ and probabilities of choosing these opinions given by equations (10)-(11). Please note that these 'colours' are equally distanced to each other and none of them is better or worse than others. Thus our scale of opinions corresponds to the 
(a) $K=3$

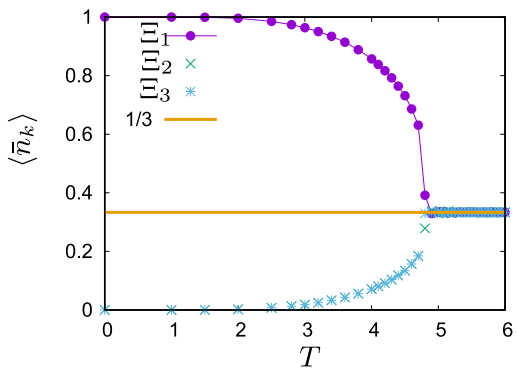

(d) $K=3$

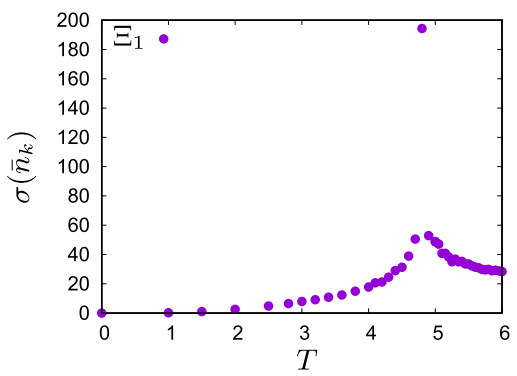

(g) $K=3$

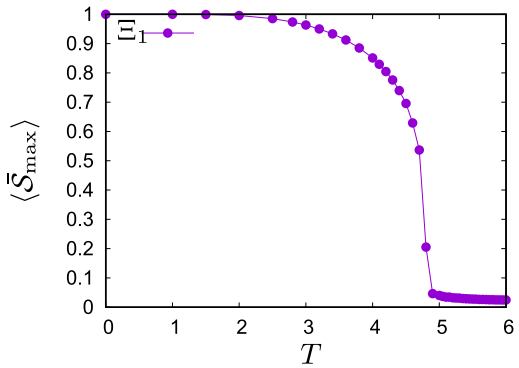

(j) $K=3$

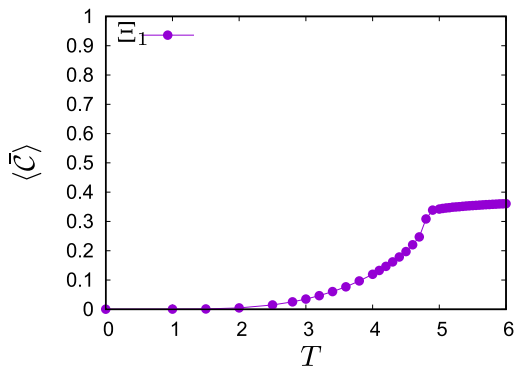

(b) $K=4$

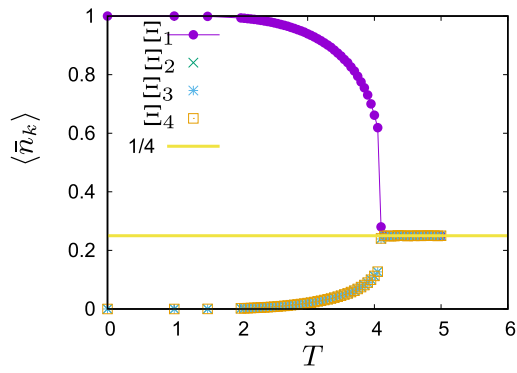

(e) $K=4$

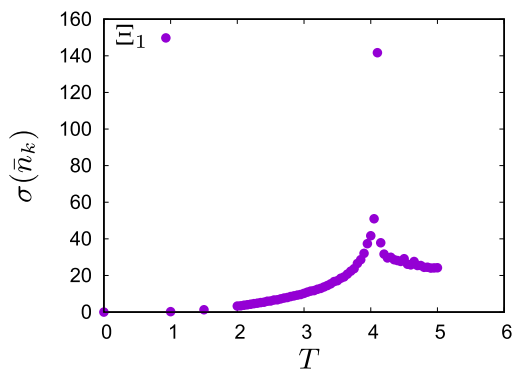

(h) $K=4$

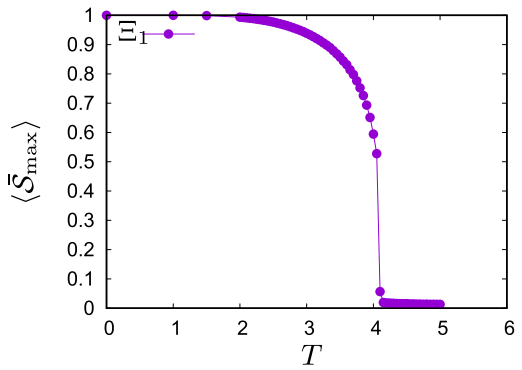

(k) $K=4$

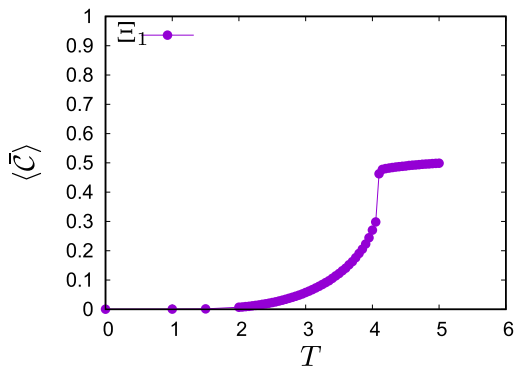

(c) $K=5$

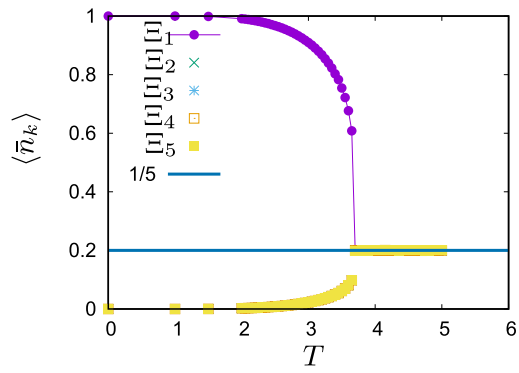

(f) $K=5$

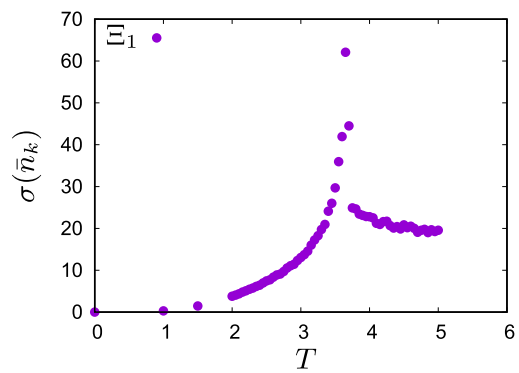

(i) $K=5$

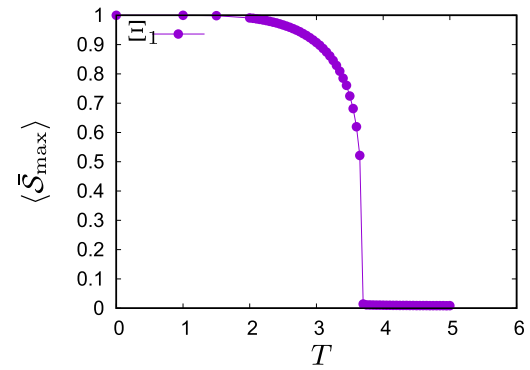

(l) $K=5$

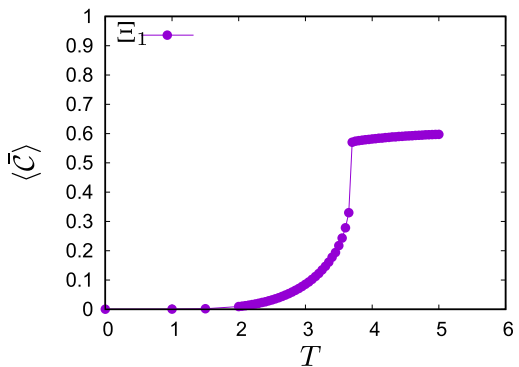

Fig. 8. The values of $(\mathrm{a}-\mathrm{c})\left\langle\bar{n}_{k}\right\rangle,(\mathrm{d}-\mathrm{f})\left\langle\sigma\left(\bar{n}_{k}\right)\right\rangle,(\mathrm{g}-\mathrm{i})\left\langle\overline{\mathcal{S}}_{\text {max }}\right\rangle$ and $(\mathrm{j}-\mathrm{l})\langle\overline{\mathcal{C}}\rangle$ for $L=40$ and $\forall i: p_{i}=s_{i}=0.5$ averaged over last $\tau=5000-100$ time steps.

nominal level of measurement [74]. Please note, that term responsible for actors interactions with other actors who share the same opinions [Eq. (8a)] is not dissimilar to the Potts model [75], where phase transition is also observed.

As we do not assign numerical values $\xi_{i}$ to differentiate actors opinions we can observe the order/disorder phase transition in thermal dependence of $\left\langle\bar{n}_{k}\right\rangle, \sigma\left(\bar{n}_{k}\right)$, $\left\langle\overline{\mathcal{S}}_{\max }\right\rangle,\langle\overline{\mathcal{C}}\rangle$. The results of our simulations indicate that the critical temperature $T_{C}$ decreases with increasing the number of opinions $K$ available in the system (see Tab. 1 and Fig. 9). We conclude, that for opinion
Table 1. The values of critical social temperature $T_{C}$ for various number $K$ of opinion available in the system deduced from Figures 6 and 8 .

\begin{tabular}{lllll}
\hline$K$ & 2 & 3 & 4 & 5 \\
\hline$T_{C}$ & 6.1 & 4.7 & 4.1 & 3.6 \\
\hline
\end{tabular}

Nowak-Szamrej-Latané model - with multi-choice of opinions and long-rage interactions among actors - the phase transition from ordered to disordered phase is also observed. 


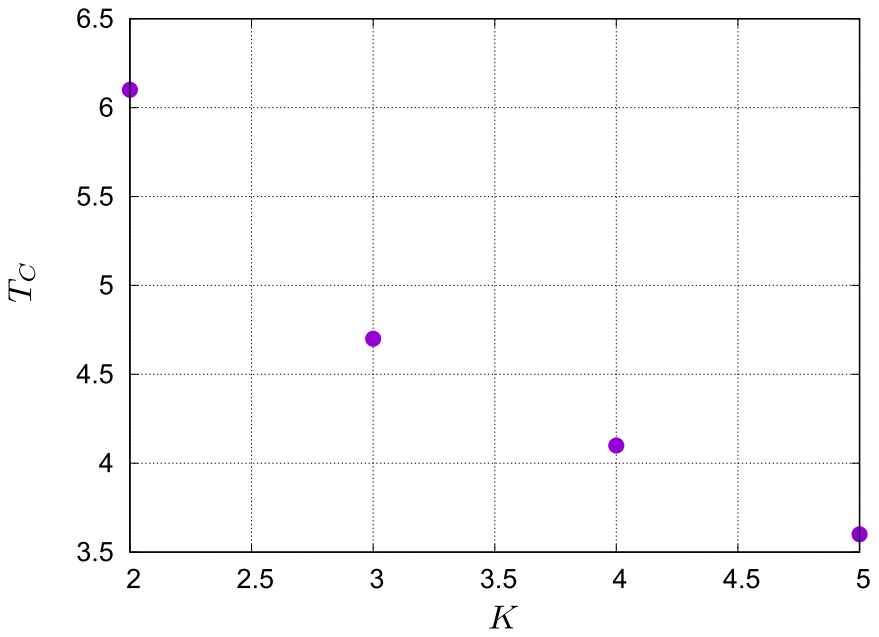

Fig. 9. Dependence of the critical social temperature $T_{C}$ on the number of available opinions $K$.

\section{Author contribution statement}

P. Bańcerowski and K. Malarz conceived the study and analysed the results. P. Bańcerowski performed the simulation. K. Malarz wrote the manuscript.

Open Access This is an open access article distributed under the terms of the Creative Commons Attribution License (http://creativecommons.org/licenses/by/4.0), which permits unrestricted use, distribution, and reproduction in any medium, provided the original work is properly cited.

\section{Appendix A: Manual}

Application was designed to show dynamic process of opinion formation. It allows to change parameters during simulation and tracking results. Application consists of two main elements:

- control and results panels on the left,

- simulation area on the right.

Control panels are divided into small windows. Each of them may be collapsed by clicking on the upper bar. From the top there are the following panels:

- Control allows to pause and restart simulation with applied parameters.

- Grid parameters contains following parameters:

Height and Width dimensions of the grid.

Number of opinion $K$, number of available opinions.

Random opinion indicates whether starting opinion of each actor is randomly chosen from $K$ allowed values or all actors have the same opinion.

Random parameters when this option is selected then parameters $p_{i}$ and $s_{i}$ are randomly chosen from range $[0,1]$ with uniform distribution.

Parameter p, Parameter s when Random parameters is not selected then all actors have the same fixed persuasiveness and supportiveness equal to this two values.

Parameters located here can not be changed during simulation. After change user have to apply them by clicking button on bottom, after this new grid will be created and previous simulation will be lost.

- Simulation parameters allows to change following parameters during the simulation:

External field button which opens pop-up with $K$ numeric values. Changing these values introduce impact from outside. It allows to strengthen or weaken a particular opinion.

Temperature social temperature $T$.

Distance function exponent exponent $\alpha$ used in distance scaling function (13).

Delay slider specifying pause time between steps of simulation.

- Leader parameters allows to manually changing opinion and parameters $p_{i}, s_{i}$ of an actor in grid centre. This allows to introduce a strong leader with persuasiveness and supportiveness higher than 1.

- Results numbers of actors with particular opinion.

- Chart dynamically generated chart which shows the results over time.

The simulation area is built of squares. Each square represents one actor. The colour of square represents opinion. Height of the darker bars on the bottom of the square indicates values of parameters $p_{i}$ (on the left) and $s_{i}$ (on the right). When simulation is paused user can click on actor to see impacts from each opinion and chance of changing opinion in next step.

\section{References}

1. D. Stauffer, in Encyclopedia of Complexity and Systems Science, edited by R.A. Meyers (Springer, New York, NY, 2009), p. 6380

2. S. Galam, Int. J. Mod. Phys. C 19, 409 (2008)

3. S. Galam, Physica A 336, 49 (2004)

4. C. Gros, Eur. Phys. J. B 90, 223 (2017)

5. P. Nyczka, K. Sznajd-Weron, J. Stat. Phys. 151, 174 (2013)

6. K. Kułakowski, Physica A 388, 469 (2009)

7. S. Gekle, L. Peliti, S. Galam, Eur. Phys. J. B 45, 569 (2005)

8. K. Sznajd-Weron, J. Sznajd, Physica A 351, 593 (2005)

9. F. Amblard, G. Deffuant, Physica A 343, 725 (2004)

10. J.A. Hołyst, K. Kacperski, F. Schweitzer, Physica A 285 , 199 (2000)

11. K. Kacperski, J.A. Hołyst, Physica A 287, 631 (2000)

12. J.-D. Mathias, S. Huet, G. Deffuant, J. Artif. Soc. Soc. Simul. 19(1), 6 (2016)

13. K. Malarz, P. Gronek, K. Kułakowski, J. Artif. Soc. Soc. Simul. 14(1), 2 (2011)

14. G. Deffuant, J. Artif. Soc. Soc. Simul. 9(3), 8 (2006)

15. R. Hegselmann, U. Krause, J. Artif. Soc. Soc. Simul. 5(3), $2(2002)$

16. T. Feliciani, A. Flache, J. Tolsma, J. Artif. Soc. Soc. Simul. 20(2), 6 (2017)

17. J. Li, R. Xiao, J. Artif. Soc. Soc. Simul. 20(2), 4 (2017)

18. P. Duggins, J. Artif. Soc. Soc. Simul. 20(1), 13 (2017) 
19. W. Lenz, Phys. Z. 21, 613 (1920)

20. E. Ising, Phys. Z. 31, 253 (1925)

21. K. Malarz, K. Kułakowski, Acta Phys. Pol. A 114, 581 (2008)

22. F. Slanina, K. Sznajd-Weron, P. Przybyla, Europhys. Lett. 82, 18006 (2008)

23. K. Sznajd-Weron, Acta Phys. Pol. B 36, 2537 (2005)

24. K. Sznajd-Weron, J. Sznajd, Int. J. Mod. Phys. C 11, 1157 (2000)

25. K. Malarz, K. Kułakowski, Acta Phys. Pol. A 117, 695 (2010)

26. G. Deffuant, D. Neau, F. Amblard, G. Weisbuch, Adv. Complex Syst. 3, 87 (2000)

27. F.W.S. Lima, Front. Phys. 5, 47 (2017)

28. K. Malarz, Int. J. Mod. Phys. C 17, 1521 (2006)

29. F. Baccelli, A. Chatterjee, S. Vishwanath, IEEE Trans. Autom. Control. 62, 5678 (2017)

30. W. Su, G. Chen, Y. Hong, Automatica 85, 448 (2017)

31. Y. Zhu, Q.A. Wang, W. Li, X. Cai, J. Stat. Mech. Theory Exp. 2017, 093401 (2017)

32. C. Anteneodo, N. Crokidakis, Phys. Rev. E 95, 042308 (2017)

33. G. Chen, H. Cheng, C. Huang, W. Han, Q. Dai, H. Li, J. Yang, Phys. Rev. E 95, 042118 (2017)

34. Y. Zhang, Q. Liu, S. Zhang, PLoS ONE 12, e0172982 (2017)

35. K. Malarz, K. Kułakowski, Europhys. News 45, 21 (2014)

36. K. Malarz, K. Kułakowski, Acta Phys. Pol. A 121, B86 (2012)

37. F.W.S. Lima, N. Crokidakis, Int. J. Mod. Phys. C 28, $1750123(2017)$

38. Y. Li, H. Cao, G. Wen, Simulation 93, 899 (2017)

39. H. Alatas, S. Nurhimawan, F. Asmat, H. Hardhienata, Chaos Solitons Fractals 101, 24 (2017)

40. M.G. Medina-Guevara, J.E. Macias-Diaz, A. Gallegos, H. Vargas-Rodriguez, Int. J. Mod. Phys. C 28, 1750058 (2017)

41. S.E. Parsegov, A.V. Proskurnikov, R. Tempo, N.E. Friedkin, IEEE Trans. Autom. Control 62, 2270 (2017)

42. G. Albi, L. Pareschi, M. Zanella, Kinet. Relat. Mod. 10, 1 (2017)

43. F. Xiong, Y. Liu, J. Cheng, Commun. Nonlinear Sci. Numer. Simul. 44, 513 (2017)

44. L.C. Evans, Partial Differential Equations, Graduate studies in mathematics, 2nd edn. (American Mathematical Society, 2010)

45. R. Hegselmann, A. Flache, V. Möller, in Tools and Techniques for Social Science Simulation, edited by R. Suleiman, K.G. Troitzsch, N. Gilbert (Physica, 2000), p. 151

46. A. Ilachinski, Cellular Automata: A Discrete Universe (World Scientific, 2001)

47. S. Wolfram, A New Kind of Science (Wolfram Media, 2002)
48. B. Chopard, M. Droz, Cellular Automata Modeling of Physical Systems (Cambridge University Press, Cambridge, 2005)

49. B. Chopard, in Computational Complexity: Theory, Techniques, and Applications, edited by R.A. Meyers (Springer, New York, NY, 2012), p. 407

50. A. Abramiuk, J. Pawłowski, K. Sznajd-Weron, Entropy 21, 521 (2019)

51. A. Jedrzejewski, K. Sznajd-Weron, C.R. Phys. (2019), 10.1016/j.crhy.2019.05.002

52. A.R. Vieira, C. Anteneodo, Phys. Rev. E 97, 052106 (2018)

53. B. Nowak, K. Sznajd-Weron, Complexity 2019, 5150825 (2019)

54. A. Jedrzejewski, G. Marcjasz, P.R. Nail, K. Sznajd-Weron, PLoS ONE 13, e0206166 (2018)

55. A. Jedrzejewski, K. Sznajd-Weron, Physica A 505, 306 (2018)

56. A. Nowak, J. Szamrej, B. Latané, Psychol. Rev. 97, 362 (1990)

57. B. Latané, S. Harkins, Percept. Psychophys. 20, 482 (1976)

58. B. Latané, S. Nida, Psychol. Bull. 89, 308 (1981)

59. B. Latané, Am. Psychol. 36, 343 (1981)

60. J.A. Hołyst, K. Kacperski, F. Schweitzer, in Annual Reviews of Computational Physics IX, edited by D. Stauffer (World Scientific, Singapore, 2011), p. 253

61. H.B. Gerard, R.A. Wilhelmy, E.S. Conolley, J. Personal. Soc. Psychol. 8, 79 (1968)

62. S.E. Asch, Sci. Am. 193, 31 (1955)

63. S. Milgram, L. Bickman, L. Berkowitz, J. Personal. Soc. Psychol. 13, 79 (1969)

64. J.M. Darley, B. Latané, J. Personal. Soc. Psychol. 8, 377 (1968)

65. M. Gansberg, "37 who saw murder didn't call the police; Apathy at stabbing of queens woman shocks inspector", New York Times (March 27, 1964), p. 1

66. R. Manning, M. Levine, A. Collins, Am. Psychol. 62, 555 (2007)

67. K. Kułakowski, arXiv:0807.0711 (2008)

68. L.M. Floría, C. Gracia-Lázaro, J. Gómez-Gardeñes, Y. Moreno, Phys. Rev. E 79, 026106 (2009)

69. M.J. de Oliveira, J. Stat. Phys. 66, 273 (1992)

70. P. Bańcerowski, Master's thesis, AGH University of Science and Technology, Kraków, 2017, in Polish

71. S.P. Anderson, A. De Palma, J.F. Thisse, Discrete Choice Theory of Product Differentiation (MIT Press, Cambridge, MA, 1992)

72. K. Byrka, A. Jedrzejewski, K. Sznajd-Weron, R. Weron, Renew. Sustain. Energy Rev. 62, 723 (2016)

73. R. Likert, Arch. Psychol. 22, 1 (1932)

74. S.S. Stevens, Science 103, 677 (1946)

75. R.B. Potts, Math. Proc. Cambridge Philos. Soc. 48, 106 (1952) 